\title{
FIBERED $p$-BISET FUNCTOR STRUCTURE OF THE FIBERED BURNSIDE RINGS
}

\author{
OLCAY COŞKUN AND DENIZ YILMAZ
}

\begin{abstract}
We determine the composition factors of the $A$-fibered Burnside functor $k B^{A}$ for $p$-groups over a field $k$ of characteristic $q$ with $q \neq p$ and cyclic fiber group $A$. We also show that, in this case, $k B^{A}$ is uniserial.
\end{abstract}

\section{INTRODUCTION}

In representation theory, it is of the utmost importance to study group actions on sets. For the simplest case, one may consider the action of a finite group $G$ on a finite set $X$. This action reveals the theory of Burnside rings. Considering the common features shared by Burnside rings and representation rings, Dress [9] and Green [11] introduced Mackey functors to give a unified treatment of these objects.

There are two ways to let two groups act on a set $X$. First, suppose that we have two groups $G$ and $H$. By considering the action of $G$ on the left and the action of $H$ on the right, we may let $G \times H$ act on $X$. In this case, the set $X$ is called a $(G, H)$ biset and this leads us to the theory of biset functors introduced by Bouc in [5]. One of the most important applications of biset functors, among many others, is the final determination of the structure of the Dade group by Bouc [7. Also in [6, Bouc and Thevénaz studied the Burnside functor of $p$-groups. They obtained that the Burnside functor of $p$-groups over a field of characteristic zero has two composition factors, one of which is the functor of torsion-free part of the Dade group and the other one is the functor of rational representations.

As a second way of letting two groups act on a set, we may consider the action of $A \times G$ on $X$ where $G$ is a finite group and $A$ is an abelian group acting on $X$ freely. Since the $A$-action is free, such an action of $A \times G$ on $X$ can be considered as $G$ acting on the $A$-fibers and in this case, the set $X$ is called an $A$-fibered $G$-set. These objects were introduced by Dress in [10] and studied by Boltje [3] and Barker [1].

In [4, Boltje and the first author combined these two notions and introduced $A$-fibered $(G, H)$-bisets. Our aim in the present paper is to extend the results of Bouc and Thevénaz on Burnside functors and to determine the composition factors of the $A$-fibered Burnside functor $k B^{A}$ of $p$-groups over a field $k$ of characteristic

Both of the authors are supported by Tübitak-1001-113F40. 
$q$ with $q \neq p$ and a cylic fiber group $A$. To be more precise, we show that in this case, the functor $k B^{A}$ is uniserial with composition factors parameterized by sets of elementary abelian $p$-groups depending only on the prime $p$ and the characteristic $q$, and not on the particular fiber group $A$.

As in the case of the (ordinary) biset functor of the Burnside functor, when $q=0$, the fibered Burnside functor $k B^{A}$ has only two composition factors. One of the factors can be identified with a subfunctor of the functor of complex characters. The other factor has the cyclic group $C_{p}$ as its minimal group, but we are unable to identify it with a natural construction.

In Section 2, we review the theory of fibered bisets and fibered biset functors from [4], together with some specializations to the case of abelian groups. In the next section, we introduce fibered Burnside rings, recall the idempotent formula for its primitive idempotents form [1] and determine the action of basic fibered bisets on these idempotents. Our main results regarding the structure of the fibered Burnside functors are contained in the last section.

\section{Fibered Bisets and Fibered Biset Functors}

In this section, we recall basic theory of fibered bisets from [4] and specialize certain results to the case of abelian groups with sufficiently large fiber groups.

2.1. Fibered bisets. Let $G$ be a finite group, $A$ be an abelian group and $X$ be a set. We call $X$ an $A$-fibered $G$-set if $X$ is an $A \times G$-set such that the action of $A$ is free with finitely many orbits. We denote by ${ }_{G}$ set $^{A}$ the category of $A$-fibered $G$ sets. Here the morphisms are given by $A \times G$-equivariant functions. The operation of disjoint union of sets induces a coproduct on ${ }_{G}$ set $^{A}$ and we denote by $B^{A}(G)$ the Grothendieck group of this category with respect to disjoint unions. The group $B^{A}(G)$ is called the $A$-fibered Burnside group and it was first introduced, in a more general way, by Dress in [10].

The basic objects in ${ }_{G} \operatorname{set}^{A}$ with respect to disjoint union are the transitive ones. We say that an $A$-fibered $G$-set is transitive provided that the $G$-action on the set of $A$-orbits is transitive. It is easy to show that there is a bijective correspondence between the isomorphism classes $[X]$ of transitive $A$-fibered $G$-sets and the $G$-conjugacy classes of pairs $(U, \phi)$ where $U$ is a subgroup of $G$ and $\phi: U \rightarrow A$ is a group homomorphism. The bijection is given by associating $X$ to $(U, \phi)$ if $U$ is the stabilizer of some $A$-orbit in $X$ and $U$ acts on this $A$-free orbit via $\phi$. We call the pair $(U, \phi)$ corresponding to $X$ the stabilizing pair of $X$. We denote by $\mathcal{M}_{G}(A)$ the set of all such pairs $(U, \phi)$, and write $[U, \phi]_{G}$ for the isomorphism class of the $A$-fibered $G$-set with the stabilizing pair $(U, \phi)$.

If $H$ is another finite group, we write ${ }_{G} \operatorname{set}_{H}^{A}$ for the category of $A$-fibered $G \times H$ sets. By the usual convention, we regard any object in this category as an $A$-fibered 
$(G, H)$-biset. We also regard any $A$-fibered biset as an operator and, in this case, we write $\left[\frac{G \times H}{U, \phi}\right]$ instead of $[U, \phi]_{G \times H}$. With this notation, any ordinary biset $\left[\frac{G \times H}{U}\right]$ is regarded as an $A$-fibered biset as $\left[\frac{G \times H}{U, 1}\right]$ where 1 denotes the trivial homomorphism sending any element of $U$ to the identity element of $A$.

Further let $K$ be another finite group, $X$ an $A$-fibered $(G, H)$-biset and $Y$ an $A$ fibered $(H, K)$-biset. We define the tensor product $X \otimes_{A H} Y$ of $X$ and $Y$ as the set of $A$-free orbits of the usual amalgamated product $X \times_{A H} Y$ of the bisets $X$ and $Y$. Recall that $X \times_{A H} Y$ is the set of $A \times H$ - orbits in $X \times Y$ under the $A \times H$-action given by

$$
(a, h) \cdot(x, y)=\left(x \cdot\left(a^{-1}, h^{-1}\right),(a, h) \cdot y\right)
$$

for any $(a, h) \in A \times H$ and $(x, y) \in X \times Y$. We denote the $A \times H$-orbit containing the pair $(x, y)$ by $\left(x, A_{A H} y\right)$. Then the group $A$ acts on the set $X \times_{A H} Y$ via $a \cdot\left(x, A_{H} y\right)=$ $\left(a \cdot x,{ }_{A H} y\right)=\left(x,{ }_{A H} a \cdot y\right)$. Given an $A$-free $A \times H$-orbit $\left(x,,_{A H} y\right)$, we denote its image in the subset $X \otimes_{A H} Y$ by $x \otimes_{A H} y$ or simply by $x \otimes y$, when there is no risk of confusion. With this notation, $X \otimes_{A H} Y$ becomes an $A$-fibered $(G, K)$-biset via

$$
(g, a) \cdot(x \otimes y) \cdot k=(g \cdot a \cdot x) \otimes(y \cdot k)
$$

for $g \in G, a \in A$ and $k \in K$. We introduce further notation to determine the product of two transitive $A$-fibered bisets.

Given a pair $(U, \phi) \in \mathcal{M}_{G \times H}(A)$, the subgroup $U$ determines the following datum: Let $P=p_{1}(U)$ and $Q=p_{2}(U)$ be the first and the second projections of $U$. Let also $K=k_{1}(U)=p_{1}(U \cap(G \times 1))$ and $L=k_{2}(U)=p_{2}(U \cap(1 \times H))$. Then we have that $K \unlhd P$ and $L \unlhd Q$. Moreover the groups $P / K$ and $Q / L$ are isomorphic and a canonical isomorphism $\eta: Q / L \rightarrow P / K$ is determined by the subgroup $U$ via $\eta(h L)=g K$ if $(g, h) \in U$. Conversely, if a quintuple $(P, K, \eta, L, Q)$ where $K \unlhd P \leq G$ and $L \unlhd Q \leq H$ and $\eta: Q / L \rightarrow P / K$ an isomorphism is given, a subgroup $U$ with the given invariants is uniquely determined by $U=\{(g, h) \in P \times Q \mid \eta(h L)=\eta(g K)\}$. This is known as Goursat's Theorem. We further write $\left.\phi\right|_{K \times L}=\phi_{1} \times \phi_{2}^{-1}$.

With this notation, if both $X$ and $Y$ are transitive, say $X=\left[\frac{G \times H}{U, \phi}\right]$ and $Y=$ $\left[\frac{H \times K}{V, \psi}\right]$, then by [4, Corollary 2.5], the above tensor product becomes

$$
\left[\frac{G \times H}{U, \phi}\right] \otimes_{A H}\left[\frac{H \times K}{V, \psi}\right]=\sum_{\substack{\left.x \in\left[p_{2}(U) \backslash H / p_{1}(V)\right] \\ \phi_{2}\right|_{H_{x}}=\left.\psi_{1}\right|_{H_{x}}}}\left[\frac{G \times K}{U *{ }^{(x, 1)} V, \phi *{ }^{(x, 1)} \psi}\right]
$$

where $H_{x}=k_{2}(U) \cap{ }^{x} k_{1}(V)$, the subgroup $U * V$ is the composition

$$
U * V=\{(g, k) \in G \times K \mid(g, h) \in U,(h, k) \in V \text { for some } h \in H\}
$$

and the homomorphism $\phi * \psi: U * V \rightarrow A$ is defined by

$$
(\phi * \psi)(g, k)=\phi(g, h) \cdot \psi(h, k)
$$


for some choice of $h \in H$ such that $(g, h) \in U$ and $(h, k) \in V$. Note that the homomorphism $\phi * \psi$ is independent of the choice of $h \in H$. The equation (1I) is sometimes referred as the Mackey product formula.

2.2. Decompositions for abelian groups. This product allows us to decompose any $A$-fibered $(G, H)$-biset into basic ones, as in the case of ordinary bisets. We refer to [4] for further details. In this paper, we only need the decomposition of fibered bisets for abelian groups with sufficiently large fiber group $A$, which we discuss next. First, we introduce the notation for basic fibered bisets which is used throughout the paper. Let $H$ be a subgroup of $G$ and $N$ be a normal subgroup of $G$. Also let $G^{\prime}$ be another finite group with a group isomorphism $\lambda: G \rightarrow G^{\prime}$.

Following Bouc [8], we define the induction from $H$ to $G$ and restriction from $G$ to $H$ as the transitive bisets

$$
\operatorname{Ind}_{H}^{G}:={ }_{G} G_{H}, \quad \operatorname{Res}_{H}^{G}:={ }_{H} G_{G}
$$

where we regard the set $G$ as a $(G, H)$-biset (resp. as an $(H, G)$-biset) in the usual way, via left and right multiplication by the corresponding group. We also define deflation from $G$ to $G / N$ and inflation from $G / N$ to $G$ as the transitive bisets

$$
\operatorname{Def}_{G / N}^{G}:={ }_{G / N}(G / N)_{G}, \quad \operatorname{Inf}_{G / N}^{G}:={ }_{G}(G / N)_{G / N} .
$$

As above, we regard the set $G / N$ as a $(G / N, G)$-biset (and as a $(G, G / N)$-biset) in the usual way. Finally, we define the transport of structure from $G^{\prime}$ to $G$ through $\lambda$ as the biset

$$
\mathrm{c}_{G, G^{\prime}}^{\lambda}:={ }_{G} G_{G^{\prime}}
$$

where the $G$-action is the left multiplication and the $G^{\prime}$-action is multiplication through $\lambda$. In all these cases, the $A$-action is trivial.

Another basic fibered biset that we need in this paper is the twist biset defined as follows. Let $\phi \in G^{*}=\operatorname{Hom}(G, A)$ be a homomorphism from $G$ to $A$. Then the twist by $\phi$ at $G$ is the $A$-fibered $(G, G)$-biset

$$
\mathrm{Tw}_{G}^{\phi}=\left(\frac{G \times G}{\Delta(G), \Delta(\phi)}\right) \text {. }
$$

Here, for any pair $(G, \phi) \in \mathcal{M}_{G}(A)$, the diagonal inclusion $(\Delta(G), \Delta(\phi))$ of $(G, \phi)$ in $\mathcal{M}_{G \times G}(A)$ is the pair consisting of the diagonal inclusion of the group $G$ in $G \times G$ and the diagonal homomorphism $\Delta(\phi)$ given by $\Delta(\phi)(g, g)=\phi(g)$.

Now let $G$ be a finite abelian group and $A$ be an abelian group. We say that $A$ is splitting for $G$ if $A$ contains an element of order $\exp (G)$. Note that, in this case, homomorphisms $G \rightarrow A$ can be identified with homomorphisms $G \rightarrow \mathbb{C}$. For the next theorem, we let $G$ and $H$ be abelian groups and $A$ be splitting for both $G$ and $H$. Then given a pair $(U, \phi) \in \mathcal{M}_{G \times H}(A)$, we write $(P, K, \eta, Q, L)$ for the invariants $\left(p_{1}(U), k_{1}(U), \eta, p_{2}(U), k_{2}(U)\right)$ where $\eta$ is the canonical isomorphism between $P / K$ 
FIBERED $p$-BISET FUNCTOR STRUCTURE OF THE FIBERED BURNSIDE RINGS

and $Q / L$ determined by $U$. We also write $\tilde{\phi}=\tilde{\phi}_{1} \times \tilde{\phi}_{2}$ for an extension of $\phi$ to $P \times Q$ which exists by the above assumption on $A$.

Theorem 2.1. Assume the above notation. Then there is an isomorphism of $A$ fibered $(G, H)$-bisets

$$
\left(\frac{G \times H}{U, \phi}\right) \cong \operatorname{Ind}_{P}^{G} \operatorname{Tw}_{P}^{\tilde{\phi}_{1}} \operatorname{Inf}_{P / K}^{P} \mathrm{c}_{P / K, Q / L}^{\eta} \operatorname{Def}_{Q / L}^{Q} \operatorname{Tw}_{Q}^{\tilde{\phi}_{2}} \operatorname{Res}_{Q}^{H} .
$$

Proof. We evaluate the product on the right hand side. Note that, in the above product, the stabilizer for $\mathrm{Tw}_{P}^{\tilde{\phi}_{1}}$ is $\Delta(P)$ and that for $\mathrm{Tw}_{Q}^{\tilde{\phi}_{2}}$ is $\Delta(Q)$. Also, it is clear from its definition that for any subgroups $V \leq G \times P$ and $V^{\prime} \leq Q \times H$, we have $V * \Delta(P)=V$ and $\Delta(Q) * V^{\prime}=V^{\prime}$. Thus, the $*$-product of the stabilizers on the right hand side gives the subgroup $U$, as in the case of ordinary bisets given in Lemma 2.3.26 in [8]. Thus we only need to check that $\phi=\left(1 *\left(\tilde{\phi}_{1} * 1\right)\right) *\left(\left(1 * \tilde{\phi}_{2}\right) * 1\right)$. Let $(g, h) \in U$. Then we have

$$
\begin{aligned}
\left(\left(1 *\left(\tilde{\phi}_{1} * 1\right)\right) *\left(\left(1 * \tilde{\phi}_{2}\right) * 1\right)\right)(g, h) & =\left(1 *\left(\tilde{\phi}_{1} * 1\right)\right)(g, g K) \cdot\left(\left(1 * \tilde{\phi}_{2}\right) * 1\right)(h L, h) \\
& =\tilde{\phi}_{1}(g) \cdot \tilde{\phi}_{2}(h)=\left(\tilde{\phi}_{1} \times \tilde{\phi}_{2}\right)(g, h)=\phi(g, h)
\end{aligned}
$$

which completes the proof of the theorem.

Remark 2.1. Suppose A satisfies Hypothesis 10.1 in [4], so that torA is divisible. Then the above condition on $A$ is satisfied trivially and hence the above theorem holds in this case. Note that [4, Theorem 10.14] describes the decomposition of an A-fibered $(G, H)$-biset for any finite groups $G$ and $H$ with the fiber group $A$ satisfying the hypothesis.

Remark 2.2. Our main interest in this paper is the case where $A$ is a finite nontrivial cyclic p-group for a prime $p$ and both $G$ and $H$ are elementary abelian p-groups. Clearly, the above theorem also holds in this case.

2.3. Fibered Biset Functors. Let $A$ be an abelian group and $R$ be a commutative ring with unity. Let $\mathcal{C}:=\mathcal{C}_{R}^{A}$ denote the following category. The objects of $\mathcal{C}$ are all finite groups. Given two finite groups $G$ and $H$, we define

$$
\operatorname{Hom}_{\mathcal{C}}(G, H):=R \otimes B(H \times G, A) .
$$

The composition is the $R$-linear extension of the tensor product of $A$-fibered bisets introduced above.

Now an $A$-fibered biset functor over $R$ is an $R$-linear functor $\mathcal{C} \rightarrow{ }_{R}$ Mod. The class of all $A$-fibered biset functors together with natural transformations between them form a category, denoted by $\mathcal{F}:=\mathcal{F}_{R}^{A}$. Since ${ }_{R}$ Mod is an abelian category, the category $\mathcal{F}$ is also abelian. By the general theory for simple functors in such categories, see [8, Section 2], simple fibered biset functors can be parameterized by 
their evaluations at groups which are of minimal order having non-zero evaluation. Explicit classification of simple fibered biset functors over a field is done in [4, Section 9]. In this paper, we only need a special case of this parametrization, where the group $G$ is an elementary abelian $p$-group for a prime number $p$. It turns out that our techniques are also valid for functors parameterized by abelian groups. Next we consider this special case for completeness.

2.4. Minimal evaluations of simple fibered biset functors for abelian groups. In this section, we consider simple fibered biset functors with minimal non-zero evaluations at abelian groups. Let $G$ be a finite group and $F$ be a fibered biset functor. Also let $E_{G}$ denote the endomorphism $\operatorname{ring}$ of $G$ in $\mathcal{C}$. Clearly, the evaluation $F(G)$ is an $E_{G}$-module. Furthermore, if $F$ is simple, then $F(G)$ is a module for the quotient algebra $\bar{E}_{G}=E_{G} / I_{G}$. Here $I_{G}$ denote the ideal generated by elements in $E_{G}$ which factor through a group of smaller order. The structure of $\bar{E}_{G}$ is described in [4, Section 8]. It turns out that when $G$ is abelian, its structure is simpler, as we describe below.

For the rest of this section, let $G$ be a finite abelian group and $A$ be an abelian group which is splitting for $G$. We first describe the structure of $\bar{E}_{G}$. Note that a similar result in the case where $A$ is cyclic of prime order and $G$ is arbitrary can be found in Lemma 15 of [12].

Let $G^{*} \rtimes \operatorname{Out}(G)$ denote the semidirect product of the groups $G^{*}$ and $\operatorname{Out}(G)$ where $\operatorname{Out}(G)$ acts on $G^{*}$ via composition, that is, $(\lambda \cdot \phi)(g)=\phi(\lambda(g))$ for any $g \in G, \phi \in G^{*}$ and $\lambda \in \operatorname{Out}(G)$. With this notation, we have the following result.

Theorem 2.2. Let $G$ be a finite abelian group and $A$ be an abelian group which is splitting for $G$. Then there is an isomorphism of $R$-algebras

$$
\bar{E}_{G} \cong R\left[G^{*} \rtimes \operatorname{Out}(G)\right] .
$$

Proof. Let $X=\left(\frac{G \times G}{U, \phi}\right)$ be a transitive $A$-fibered $(G, G)$-biset. With the notation of Theorem 2.1, we can write

$$
\left(\frac{G \times H}{U, \phi}\right) \cong \operatorname{Ind}_{P}^{G} \operatorname{Tw}_{P}^{\tilde{\phi}_{1}} \operatorname{Inf}_{P / K}^{P} \mathrm{c}_{P / K, Q / L}^{\eta} \operatorname{Def}_{Q / L}^{Q} \operatorname{Tw}_{Q}^{\tilde{\phi}_{2}} \operatorname{Res}_{Q}^{H} .
$$

By this isomorphism, it is clear that $X$ is in the ideal $I_{G}$ unless $P=Q=G$ and $K=L=1$. Therefore the quotient $\bar{E}_{G}$ can be identified with the submodule of $E_{G}$ generated by all $A$-fibered $(G, G)$-bisets $X=\left(\frac{G \times G}{U, \phi}\right)$ where

$$
P=Q=G, \quad K=L=1, \quad U=\{(g, \lambda(g)) \in G \times G \mid \lambda \in \operatorname{Aut}(G)\} .
$$

Thus by Theorem 2.1, we get

$$
\left(\frac{G \times G}{U, \phi}\right) \cong \operatorname{Tw}_{G}^{\tilde{\phi}_{1}} \otimes_{A G} \mathrm{c}_{G, G}^{\lambda} \otimes \operatorname{Tw}_{G}^{\tilde{\phi}_{2}}
$$


where $\tilde{\phi}=\tilde{\phi}_{1} \times \tilde{\phi}_{2}$ is an extension of $\phi$ to $G \times G$ and $\lambda \in \operatorname{Aut}(G)$. It follows from the Mackey product formula that if $g \in G$ and $\mathrm{c}_{G, G}^{g}$ denotes the inner automorphism of $G$ induced by conjugation with $g$, then

$$
\mathrm{c}_{G, G}^{g} \otimes_{A G}\left(\frac{G \times G}{U, \phi}\right) \cong\left(\frac{G \times G}{U, \phi}\right) \otimes_{A G} \mathrm{c}_{G, G}^{g} \cong\left(\frac{G \times G}{U, \phi}\right) .
$$

Hence, up to isomorphism, the automorphism $\lambda: G \rightarrow G$ can be taken as an outer automorphism. Furthermore, we have

$$
\mathrm{c}_{G, G}^{\lambda} \otimes_{A G} \mathrm{Tw}_{G}^{\phi} \cong \mathrm{Tw}_{G}^{\phi \circ \lambda} \otimes_{A G} \mathrm{c}_{G, G}^{\lambda}
$$

for any $\phi \in G^{*}$ and $\lambda \in \operatorname{Out}(G)$. Thus the algebra $\bar{E}_{G}$ is generated by all $A$ fibered $(G, G)$-bisets of the form $\operatorname{Tw}_{G}^{\phi} \otimes_{A G} \mathrm{c}_{G, G}^{\lambda}$ where $\phi \in G^{*}$ and $\lambda \in \operatorname{Out}(G)$. For simplicity, we put

$$
[\phi, \lambda]_{G}:=\operatorname{Tw}_{G}^{\phi} \otimes_{A G} \mathrm{c}_{G, G}^{\lambda}
$$

Now we define

$$
\alpha: \bar{E}_{G} \rightarrow R\left[G^{*} \rtimes \operatorname{Out}(G)\right]
$$

by $\alpha\left([\phi, \lambda]_{G}\right)=(\phi, \lambda)$. Clearly the linear extension of $\alpha$ is a well-defined isomorphism of abelian groups. We only show that it induces an algebra map. Given $[\phi, \lambda]_{G},\left[\phi^{\prime}, \lambda^{\prime}\right]_{G} \in \bar{E}_{G}$, we have

$$
\alpha\left([\phi, \lambda]_{G}\right) \cdot \alpha\left(\left[\phi^{\prime}, \lambda^{\prime}\right]_{G}\right)=(\phi, \lambda) \cdot\left(\phi^{\prime}, \lambda^{\prime}\right)=\left(\phi \cdot\left(\lambda \cdot \phi^{\prime}\right), \lambda \lambda^{\prime}\right)
$$

by definition of the semidirect product. On the other hand, by the Mackey product formula, we have

$$
[\phi, \lambda] \cdot\left[\phi^{\prime}, \lambda^{\prime}\right]=\left[\phi \cdot\left(\lambda \cdot \phi^{\prime}\right), \lambda \lambda^{\prime}\right]_{G}
$$

as required.

Now let $k$ be a field and $S$ be a simple fibered biset functor over $k$ with minimal group $G$. Clearly the evaluation $V=S(G)$ is a simple $\bar{E}_{G}$-module. Hence, by the previous theorem, $V$ is a simple $k\left[G^{*} \rtimes \operatorname{Out}(G)\right]$-module. By the general theory

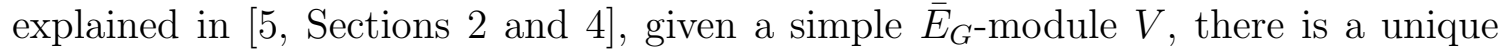
simple fibered biset functor $S$ such that $S(G)=V$. We denote the simple functor corresponding to $V$ by $S_{G, V}$. Note that, in general, a simple fibered biset functor may have two non-isomorphic minimal groups. Thus at this point, we do not know if the simple functor $S_{G, V}$ has another minimal group when $G$ is abelian. For the aims of this paper, this is not a problem. We remark that the exact situation can be determined using several results from [4]. 


\section{Fibered Burnside Functor}

In this section, we recall the ring structure on the fibered Burnside group from [1, and introduce a natural fibered biset functor structure on it. We also determine the actions of basic fibered bisets on primitive idempotents of the fibered Burnside ring.

3.1. The ring structure. Let $G$ be a finite group and $A$ be an abelian group. The $A$-fibered Burnside group $B^{A}(G)$ can be identified with the free abelian group

$$
B^{A}(G)=\bigoplus_{[U, \phi]_{G} \in \mathcal{M}_{G}(A) / G} \mathbb{Z} \cdot[U, \phi]_{G}
$$

on the set of $G$-conjugacy classes of pairs $(U, \phi) \in \mathcal{M}_{G}(A)$. Following [10], we make $B^{A}(G)$ a ring via the linear extension of the following product. For $A$-fibered $G$-sets $X$ and $Y$, we define $X \cdot Y$ to be the union of $A$-orbits of $X \times Y$ with respect to the $A$-action

$$
a \cdot(x, y)=\left(a \cdot x, a^{-1} \cdot y\right)
$$

for $a \in A$ and $(x, y) \in X \times Y$. We denote the $A$-orbit containing the pair $(x, y)$ by $\left(x,{ }_{A} y\right)$ and make the set $X \cdot Y$ an $A$-fibered $G$-set via $(g, a) \cdot\left(x,{ }_{A} y\right)=\left(g \cdot a \cdot x,_{A} g \cdot y\right)$ for $(g, a) \in G \times A$ and $(x, A y) \in X \cdot Y$. By [1, Remark 2.3] and [3, 5.3], for $[U, \phi]_{G}$ and $[V, \psi]_{G}$ in $B^{A}(G)$, we have

$$
[U, \phi]_{G} \cdot[V, \psi]_{G}=\sum_{t \in[U \backslash G / V]}\left[U \cap{ }^{t} V, \phi \cdot{ }^{t} \psi\right]_{G} .
$$

The ring $B^{A}(G)$ is commutative and unital with unit $[G, 1]_{G}$ and it extends the (ordinary) Burnside ring $B(G)$. Here we identify $B(G)$ with the subring of $B^{A}(G)$ generated by all the elements $[H, 1]_{G}$ as $H$ runs over all subgroups of $G$. Moreover, following [8, Remark 2.5.7], we can identify $B^{A}(G)$ with a subring of the $A$-fibered double Burnside ring $B(G \times G, A)$ as follows. Note that the ring structure on $B(G \times$ $G, A)$ comes from the composition product of $A$-fibered $(G, G)$-bisets.

For an $A$-fibered $G$-set $X$, we define $A$-fibered $(G, G)$-biset $\Delta(X):=G \times X$ where the $A$-action is given by

$$
a \cdot(g, x)=(g, a \cdot x)
$$

for any $a \in A$ and $(g, x) \in G \times X$ and the $(G, G)$-action is given by

$$
g_{1} \cdot(g, x) \cdot g_{2}=\left(g_{1} \cdot g \cdot g_{2}, g_{2}^{-1} \cdot x\right)
$$

for any $g, g_{1}, g_{2} \in G$ and $x \in X$. Note that for a transitive $A$-fibered $G$-set $[U, \phi]_{G}$, the above definition becomes

$$
\Delta([U, \phi])=\left[\frac{G \times G}{\Delta(U), \Delta(\phi)}\right] .
$$


Here, for any pair $(U, \phi) \in \mathcal{M}_{G}(A)$, the diagonal inclusion $(\Delta(U), \Delta(\phi))$ of $(U, \phi)$ in $\mathcal{M}_{G \times G}(A)$ is the pair consisting of the diagonal inclusion of the subgroup $U$ in $G \times G$ and the diagonal homomorphism $\Delta(\phi)$ given by $\Delta(\phi)(u, u)=\phi(u)$. Extending this map linearly to the $A$-fibered Burnside group $B^{A}(G)$, we get a group homomorphism

$$
\Delta: B^{A}(G) \longrightarrow B^{A}(G \times G)
$$

Note that the map $\Delta$ extends the map

$$
\Delta: B(G) \rightarrow B(G, G)
$$

defined in [8, Lemma 2.5.8]. Here $B(G)$ (resp. $B(G, G)$ ) denotes the Burnside ring (resp. the double Burnside ring) of the group $G$. Moreover we have the following result.

Proposition 3.1. The map $\Delta$ is a unital ring homomorphism where $B^{A}(G \times G)$ is considered as a ring with the composition of fibered bisets.

Proof. It follows from the definition that the map $\Delta$ is a group homomorphism. We only prove that it is unital and it preserves products, that is, we prove that $\Delta$ is unital and for any $A$-fibered $G$-sets $X$ and $Y$, there is an isomorphism

$$
\Delta(X \cdot Y) \cong \Delta(X) \otimes_{A G} \Delta(Y)
$$

of $A$-fibered $(G, G)$-bisets. Since $\Delta$ extends a similar map from the Burnside ring of $G$ to the double Burnside ring of $G$, it is unital. Next, we let $X$ and $Y$ be $A$-fibered $G$-sets. Note that, by definition of $\Delta$, the $A \times G \times G$-set $\Delta(X) \times{ }_{A G} \Delta(Y)$ is $A$-free and hence we have $\Delta(X) \times_{A G} \Delta(Y)=\Delta(X) \otimes_{A G} \Delta(Y)$ as $A$-fibered $(G, G)$-bisets. Now we define

$$
\beta: \Delta(X \cdot Y) \rightarrow \Delta(X) \otimes_{A G} \Delta(Y)
$$

by $\beta\left(\left(g,\left(x,{ }_{A} y\right)\right)\right)=(g, x) \otimes(1, y)$. It is straightforward to show that $\beta$ is independent from the choice of the $A$-orbit representative $(x, y)$ of $\left(x,{ }_{A} y\right)$. Moreover $\beta$ commutes with the $A \times G \times G$-action. Indeed, if $a \in A$ and $g_{1}, g_{2} \in G$, then

$$
\begin{aligned}
\beta\left(\left(a, g_{1}\right) \cdot\left(g,\left(x,{ }_{A} y\right)\right) \cdot g_{2}\right) & =\beta\left(\left(g_{1} g g_{2}, a \cdot\left(x,{ }_{A} y\right) \cdot g_{2}\right)\right)=\beta\left(\left(g_{1} g g_{2},\left(a \cdot g_{2}^{-1} \cdot x,{ }_{A} g_{2}^{-1} \cdot y\right)\right)\right) \\
& =\left(g_{1} g g_{2}, a \cdot g_{2}^{-1} \cdot x\right) \otimes\left(1, g_{2}^{-1} \cdot y\right) \\
& =\left(\left(g_{1}, a\right) \cdot(g, x) \cdot g_{2}\right) \otimes\left(1, g_{2}^{-1} \cdot y\right) \\
& =\left(\left(a, g_{1}\right) \cdot(g, x)\right) \otimes\left(g_{2}, g_{2}^{-1} \cdot y\right) \\
& =\left(\left(a, g_{1}\right) \cdot(g, x)\right) \otimes\left((1, y) \cdot g_{2}\right) \\
& =\left(a, g_{1}\right) \cdot((g, x) \otimes(1, y)) \cdot g_{2} \\
& =\left(a, g_{1}\right) \cdot \beta\left(\left(g,\left(x,{ }_{A} y\right)\right)\right) \cdot g_{2} .
\end{aligned}
$$

Next we define the function

$$
\gamma: \Delta(X) \otimes_{A G} \Delta(Y) \rightarrow \Delta(X \cdot Y)
$$


given by $\gamma((g, x) \otimes(h, y))=\left(g h,\left(h^{-1} \cdot x,{ }_{A} y\right)\right)$. It is easy to check that $\gamma$ is an inverse to $\beta$ as a morphism of $A$-fibered $(G, G)$-bisets.

We note that the above proof is very similar to proof of the first part of Lemma 2.5.8 in [8]. With this result, we identify $B^{A}(G)$ with its image in $B(G \times G, A)$ via the map $\Delta$. Under this identification, given any $A$-fibered biset functor $F$, the evaluation $F(G)$ of $F$ at $G$ becomes a $B^{A}(G)$-module via restriction through $\Delta$.

3.2. The functorial structure. Let $k$ be a field. We denote by $k B^{A}$ the $A$-fibered biset functor over $k$ which associates a finite group $G$ to the $A$-fibered Burnside group $k B^{A}(G)=k \otimes B^{A}(G)$ and any $A$-fibered $(H, G)$-biset $X$ to the map

$$
k B^{A}(X): k B^{A}(G) \rightarrow k B^{A}(H) .
$$

given by left multiplication. To simplify the notation, we denote the map $k B^{A}(X)$ by $X$. Explicitly, if $(U, \phi) \in \mathcal{M}_{G}(A)$, then

$$
\left[\frac{H \times G}{V, \psi}\right] \cdot[U, \phi]_{G}=\sum_{\substack{x \in\left[p_{2}(V) \backslash G / U\right] \\ \psi_{2} \mid k_{2}(V) \cap U}=\left.x\right|_{k_{2}(V) \cap U}}\left[V *{ }^{x} U, \psi * *^{x} \phi\right]_{H} .
$$

The functor $k B^{A}$ can be identified with the Yoneda functor $\operatorname{Hom}_{\mathcal{C}}(-, 1)$ where 1 denotes the trivial group. In particular, it is projective and by Yoneda's Lemma, we have $\operatorname{End}_{\mathcal{F}}\left(k B^{A}\right) \cong k B^{A}(1)=k \otimes \mathbb{Z} \cong k$ as rings. In particular, the endomorphism ring of $k B^{A}$ is local, and hence the functor $k B^{A}$ is indecomposable. By the classification of simple $A$-fibered biset functors, it is the projective cover of the simple $A$-fibered biset functor $S_{1, k}$ where $k$ denotes the one dimensional $k$-vector space. The following proposition is a crucial result that we need to describe the other composition factors of $k B^{A}$.

Proposition 3.2. The evaluation $k B^{A}(G)$ of $k B^{A}$ at $G$ regarded as a module over $k B^{A}(G)$ via restriction through $\Delta$ is the regular $k B^{A}(G)$-module. In particular, for any subfunctor $F$ of $k B^{A}$ and for any finite group $G$, the evaluation $F(G)$ is an ideal of $k B^{A}(G)$.

Proof. To prove the first assertion, it suffices to prove that there is an isomorphism of $A$-fibered $G$-sets

$$
\Delta(X) \otimes_{A G} Y \cong X \cdot Y
$$

for any $A$-fibered $G$-sets $X, Y \in B^{A}(G)$. Following the proof of Lemma 2.5.8 of [8], we define

$$
\alpha: \Delta(X) \otimes_{A G} Y \rightarrow X \cdot Y
$$

by $\alpha((g, x) \otimes y)=\left(g \cdot x,{ }_{A} g \cdot y\right)$. This is well-defined with inverse

$$
\beta: X \cdot Y \rightarrow \Delta(X) \otimes_{A G} Y
$$


given by $\beta\left(\left(x,{ }_{A} y\right)\right)=(1, x) \otimes y$. We leave the details to reader and note that the proofs are almost identical to those in [8], one only needs to check the $A$-action. Now the second part of the statement follows from the first part since the evaluation $F(G)$ becomes a submodule of the regular $k B^{A}(G)$-module.

Remark 3.1. The second part of the above result holds for an arbitrary Green biset functor, that is, given a Green biset functor $A$, as in Section 8.5 of [8], an $A$ submodule $F$ of $A$ and a finite group $G$, then $F(G)$ is an ideal of $A(G)$ with the ring structure on $A(G)$ induced from the Green biset functor structure.

3.3. Actions on idempotents. With Proposition [3.2, we see that there is a connection between subfunctors of the fibered Burnside functor $k B^{A}$ and the structure of the Burnside ring $k B^{A}(G)$. To make this relation explicit, we need to determine the action of basic $A$-fibered biset operations on primitive idempotents of the ring $k B^{A}(G)$. There are two formulas for primitive idempotents of $k B^{A}(G)$, see [1] and [3]. In this paper, we use Barker's formula.

Let $A$ be a cyclic $p$-group and suppose $k$ is a sufficiently large field of characteristic 0 . Also let $O(G)=O_{A}(G)$ denote the intersection of kernels of all homomorphisms $G \rightarrow A$. In this case, the group $G^{*}$ is isomorphic with the dual group $\operatorname{Hom}(G / O(G), A)$ of the group $G / O(G)$. We denote by $\mathcal{E}_{G}(A)$ the set of all pairs $(H, h)$ where $H \leq G$ and $h$ runs over a complete set of left coset representatives of $O(H)$ in $H$. The set $\mathcal{E}_{G}(A)$ is a $G$-set via conjugation, and we have $\left|\mathcal{M}_{G}(A)\right|=\left|\mathcal{E}_{G}(A)\right|$ by [1, Lemma 3.1]. It turns out that there is a bijective correspondence between the set of primitive idempotents of $k B^{A}(G)$ and the set $\mathcal{E}_{G}(A)$. Writing $e_{H, h}^{G}$ for the primitive idempotent corresponding to the pair $(H, h)$, [1, Theorem 5.2] gives

$$
e_{H, h}^{G}=\frac{1}{\left|N_{G}(H, h)\right|} \sum_{(V, \nu) \in \mathcal{M}_{G}(A) / G}|V| \mu_{G}(V, \nu ; H, h)[V, \nu]_{G}
$$

where $N_{G}(H, h)$ denotes the stabilizer in $G$ of the pair $(H, h)$ under the above action of $G$ and where

$$
\mu_{G}(V, \nu ; H, h)=\sum_{\left(V^{\prime}, \nu^{\prime}\right) \in[V, \nu]_{G}} \nu^{\prime-1}\left(V^{\prime} \cap h O(H)\right) \mu\left(V^{\prime}, H\right) /\left|V^{\prime}\right|
$$

is the monomial Möbius function and the above sum is over all pairs $\left(V^{\prime}, \nu^{\prime}\right) G$ conjugate to $(V, \nu)$.

The idempotents $e_{H, h}^{G}$ can also be characterized using the algebra maps $k B^{A}(G) \rightarrow$ $k$, called species, introduced by Dress 10. The set of all species is known to be a basis for the dual space of the fibered Burnside ring, see [10] or [1, Lemma 5.1]. For any $(H, h) \in \mathcal{E}_{G}(A)$, we denote the species associated to the $G$-conjugacy class of 
$(H, h)$ by

$$
s_{H, h}^{G}: k B^{A}(G) \rightarrow k
$$

which is given, for an $A$-fibered $G$-set $X$, by

$$
s_{H, h}^{G}[X]=\sum_{A x} \phi_{x}(h) .
$$

Here the index $A x$ runs over the fibers of $X$ that are stabilized by $H$ and given such an orbit $A x$, the $H$-action on $A x$ induces a group homomorphism $\phi_{x}: H \rightarrow A$ given by $\phi_{x}(h)=a$ provided that $h \cdot x=a \cdot x$ for some (unique) $a \in A$. Now by the duality, the idempotent $e_{H, h}^{G} \in k B^{A}(G)$ is the unique element such that

$$
s_{K, k}^{G}\left(e_{H, h}^{G}\right)= \begin{cases}1 & (H, h)=_{G}(K, k) \\ 0 & \text { otherwise. }\end{cases}
$$

In particular, $e_{H, h}^{G}$ is the only non-zero idempotent of $k B^{A}(G)$ such that $X \cdot e_{H, h}^{G}=$ $s_{H, h}^{G}(X) e_{H, h}^{G}$ for any $X \in k B^{A}(G)$. Thus, for any $X \in k B^{A}(G)$, we have the coordinate decomposition

$$
X=\sum_{(H, h) \in \mathcal{E}_{G}(A) / G} s_{H, h}^{G}(X) e_{H, h}^{G} .
$$

Remark 3.2. Let $G$ be a p-group. The above idempotent formula still holds if we replace $k$ with a sufficiently large field of characteristic $q \neq p$ since all the denominators of the formula are p-powers and hence invertible in $k$. Thus when we restrict to the fibered p-biset functor of fibered Burnside ring over such fields, the above formula will remain valid.

Our next aim is to determine the actions of basic fibered bisets on primitive idempotents. Two of them are described by Barker in [1, Proposition 5.4 and Proposition 5.5]. We recall the result.

Proposition 3.3 (Barker). Let $K \leq G$ be finite groups. Then

(i) $\operatorname{For}(H, h) \in \mathcal{E}_{G}(A)$, we have

$$
\operatorname{Res}_{K}^{G} e_{H, h}^{G}=\sum_{(J, j)} e_{J, j}^{K}
$$

where the pairs $(J, j)$ run over representatives of the $K$-classes of the pairs which are $G$-conjugate to $(H, h)$.

(ii) Let $(H, h) \in \mathcal{E}_{K}(A)$. Then

$$
\operatorname{Ind}_{K}^{G} e_{H, h}^{K}=\left|N_{G}(H, h): N_{K}(H, h)\right| e_{H, h}^{G} .
$$


The following Frobenius type formula is used in the next theorem to describe actions of inflation and deflation.

Lemma 3.1. (Frobenius Relation) Let $N \unlhd G$ be a normal subgroup of $G$. Then, for any $A$-fibered $G$-set $X$ and any $A$-fibered $G / N$-set $Y$, we have an isomorphism of $A$-fibered $G / N$-sets

$$
Y \cdot \operatorname{Def}_{G / N}^{G}(X) \cong \operatorname{Def}_{G / N}^{G}\left(\operatorname{Inf}_{G / N}^{G}(Y) \cdot X\right)
$$

Proof. Recall that the deflation map $\operatorname{Def}_{G / N}^{G}$ (resp. the inflation map $\operatorname{Inf}_{G / N}^{G}$ ) corresponds to composition with the $(G / N, G)$-biset $G / N$ (resp. $(G, G / N)$-biset $G / N)$. Thus, given an $A$-fibered $G$-set $X$ and an $A$-fibered $G / N$-set $Y$, we have to prove

$$
Y \cdot\left(G / N \otimes_{A G} X\right) \cong G / N \otimes_{A G}\left(\left(G / N \otimes_{A(G / N)} Y\right) \cdot X\right) .
$$

Moreover by the proof of Proposition 3.2, we can rewrite the above equality as

$$
\Delta(Y) \otimes_{A(G / N)}\left(G / N \otimes_{A G} X\right) \cong G / N \otimes_{A G}\left(\Delta\left(G / N \otimes_{A(G / N)} Y\right) \otimes_{A G} X\right) .
$$

Hence by the associativity of the product $\otimes_{A G}$, it is sufficient to prove that

$$
\Delta(Y) \otimes_{A(G / N)} G / N \cong G / N \otimes_{A G} \Delta\left(G / N \otimes_{A(G / N)} Y\right)
$$

as $A$-fibered $(G / N, G)$-sets. This is a straightforward generalization of part 1 of Proposition 2.5.10 in [8]. We leave the details to reader.

Theorem 3.1. Let $N \unlhd G$ be a normal subgroup of $G$.

(i) For any $(H / N, h N) \in \mathcal{E}_{G}(A)$, we have

$$
\operatorname{Inf}_{G / N}^{G}\left(e_{H / N, h N}^{G / N}\right)=\sum_{(K, k)} e_{K, k}^{G}
$$

where $(K, k)$ runs over representatives of the $G$-classes of $\mathcal{E}_{G}(A)$ such that $(K N, k)$ is $G$-conjugate to $(H, h)$.

(ii) For any $(H, h) \in \mathcal{E}_{G}(A)$, we have

$$
\operatorname{Def}_{G / N}^{G} e_{H, h}^{G}=m_{H, h}^{G} \cdot e_{H N / N, h N}^{G / N}
$$

for some constant $m_{H, h}^{G}$.

Proof. First we demonstrate that for any $(K, k) \in \mathcal{E}_{G}(A)$ and for any $A$-fibered $G / N$ set $S$, we have $s_{K, k}^{G}\left(\operatorname{Inf}_{G / N}^{G}(S)\right)=s_{K N / N, k N}^{G / N}(S)$. Since the inflation map is a group homomorphism, it suffices to take $S$ transitive. For any transitive $A$-fibered $G / N$-set $[V / N, \nu]_{G / N}$, Equation (3) implies

$$
\operatorname{Inf}_{G / N}^{G}\left([V / N, \nu]_{G / N}\right)=G / N \otimes_{A(G / N)}[V / N, \nu]_{G / N}=[V, \bar{\nu}]_{G}
$$


where $\bar{\nu}$ denotes the inflation of $\nu$ to $V$. Thus, we have

$$
s_{K, k}^{G}\left(\operatorname{Inf}_{G / N}^{G}\left([V / N, \nu]_{G / N}\right)\right)=s_{K, k}^{G}\left([V, \bar{\nu}]_{G}\right)=\sum_{A g} \bar{\nu}_{g}(k)
$$

where $A g$ runs over the fibers stabilized by $K$. But these fibers are also stabilized by $K N / N$ and we have $\bar{\nu}_{g}(k)=\nu_{g}(k N)$. Hence

$$
s_{K, k}^{G}\left(\operatorname{Inf}_{G / N}^{G}\left([V / N, \nu]_{G / N}\right)\right)=s_{K N / N, k N}^{G / N}\left([V / N, \nu]_{G / N}\right) .
$$

Therefore, we obtain

$$
\begin{aligned}
s_{K, k}^{G}\left(\operatorname{Inf}_{G / N}^{G}\left(e_{H / N, h N}^{G / N}\right)\right) & =s_{K N / N, k N}^{G / N}\left(e_{H / N, h N}^{G / N}\right)= \begin{cases}1 & \text { if }(K N / N, k N)=_{G / N}(H / N, h N), \\
0 & \text { otherwise }\end{cases} \\
& = \begin{cases}1 & \text { if }(K N, k)=_{G}(H, h), \\
0 & \text { otherwise }\end{cases}
\end{aligned}
$$

and the first part follows.

For the second part, let $S$ be an arbitrary $A$-fibered $G / N$-set. Then, using the Frobenius relation, we obtain

$$
\begin{aligned}
S \cdot \operatorname{Def}_{G / N}^{G} e_{H, h}^{G} & =\operatorname{Def}_{G / N}^{G}\left(\operatorname{Inf}_{G / N}^{G}(S) \cdot e_{H, h}^{G}\right)=\operatorname{Def}_{G / N}^{G}\left(s_{H, h}^{G}\left(\operatorname{Inf}_{G / N}^{G} S\right) \cdot e_{H, h}^{G}\right) \\
& =\operatorname{Def}_{G / N}^{G}\left(s_{H N / N, h N}^{G / N}(S) \cdot e_{H, h}^{G}\right)=s_{H N / N, h N}^{G / N}(S) \cdot \operatorname{Def}_{G / N}^{G}\left(e_{H, h}^{G}\right) .
\end{aligned}
$$

However, $e_{H N / N, h N}^{G / N}$ is the unique element with the above property. Therefore, we conclude that

$$
\operatorname{Def}_{G / N}^{G} e_{H, h}^{G}=m \cdot e_{H N / N, h N}^{G / N}
$$

for some constant $m$.

Finally we describe the actions of transport of structure and twist bisets. We skip the straightforward proofs.

Proposition 3.4. Let $\lambda: G \rightarrow G^{\prime}$ be a group isomorphism, let $\phi \in G^{*}$ and let $(H, h) \in \mathcal{E}_{G}(A)$. Then

(i) $c_{G, G^{\prime}}^{\lambda} e_{H, h}^{G}=e_{\lambda(H), \lambda(h)}^{G^{\prime}}$,

(ii) $\mathrm{Tw}_{G}^{\phi} e_{H, h}^{G}=\phi(h) \cdot e_{H, h}^{G}$.

\section{Composition factors over p-Groups}

In this section, we restrict our attention to the category of $A$-fibered $p$-biset functors and determine the subfunctors of the fibered Burnside functor $k B^{A}$ over a field $k$ of characteristic $q \neq p$. Here by an $A$-fibered $p$-biset functor we mean a $k$-linear functor $\mathcal{C}_{p} \rightarrow{ }_{k}$ Mod where $\mathcal{C}_{p}$ is the full subcategory of $\mathcal{C}$ consisting only of $p$-groups, 
for a fixed prime $p$. We also assume that $A$ is a subgroup of the unit group of a field of characteristic zero and that the order of $A$ is divisible by $p$. Our approach is similar to that of Bouc and Thévenaz [6, Section 8].

The precise situation is as follows. We fix a prime $p$ and a positive integer $n$. We denote by $\mu_{n}$ a cyclic group of order $p^{n}$. For a $p$-group $P$, we let $O_{n}(P)$ denote the group $O_{\mu_{n}}(P)$. Finally we let $k$ be an algebraically closed field of characteristic $q \neq p$ and fix an embedding $\mu_{n} \rightarrow k^{\times}$. Our aim is to determine subfunctors of the functor $k \otimes B^{\mu_{n}}$. For simplicity write $k B^{n}:=k \otimes B^{\mu_{n}}$. For this aim, we first determine minimal groups for the subfunctors of the fibered Burnside functor.

Interestingly, in all cases, the minimal groups are elementary abelian. As in the case of the ordinary Burnside functor, extra work should be done to see which elementary abelian groups appear as a minimal group and it turns out that the possible ranks depends only on whether $q \mid p-1$.

To begin with, let $F$ be a subfunctor of $k B^{n}$ and suppose that $G$ is a minimal group of $F$. We know, by Proposition 3.2, that $F(G)$ is an ideal of $k B^{n}(G)$. Therefore, it is generated by a set of primitive idempotents $e_{H, h}^{G}$ of $k B^{n}(G)$. Let $X$ be a fibered $(L, G)$-biset for some group $L$. If $X$ can be factored through a group $K$ with $|K|<$ $|G|$, then for any $e_{H, h}^{G} \in F(G)$ we should have $X \cdot e_{H, h}^{G}=0$. This implies that to find the minimal groups, we need a deeper understanding of the action on the idempotents of the fibered bisets that map to groups of smaller order.

First, notice that for any proper subgroup $H<G$, the idempotent $e_{H, h}^{G}$ is not contained in $F(G)$. Indeed $\operatorname{Res}_{H}^{G} e_{H, h}^{G}=e_{H, h}^{H}$ and $F(H)=0$. Therefore, the ideal $F(G)$ must be generated by idempotents of the form $e_{G, g}^{G}$.

Next we consider the action of deflation maps on the idempotents of the form $e_{G, g}^{G}$. Recall that if $N \unlhd G$ is a normal subgroup of $G$, then

$$
\operatorname{Def}_{G / N}^{G} e_{G, g}^{G}=m \cdot e_{G / N, g N}^{G / N}
$$

for some constant $m$. Since $G$ is minimal, for any non-trivial normal subgroup $N$ of $G$ the constant $m$ should be zero. Following Bouc and Thevénaz [6], we consider the elementary abelian $p$-groups and non-elementary abelian $p$-groups, separately. Let $\Phi(G)$ denote the Frattini subgroup of $G$ and $\bar{G}$ denote the quotient $G / \Phi(G)$.

Lemma 4.1. For any $p$-group $G$ and $g \in G$, we have

$$
\operatorname{Def}_{\bar{G}} e_{G, g}^{G}=\frac{|O(G)|}{\left|N_{G}(G, g)\right|} \cdot|\bar{G}| \cdot e_{\bar{G}, g \Phi(G)}^{\bar{G}} .
$$

Proof. Recall the idempotent formula

$$
e_{G, g}^{G}=\frac{1}{\left|N_{G}(G, g)\right|} \sum_{(V, \nu) \in_{G} \mathcal{M}_{G}(A)}|V| \mu_{G}(V, \nu ; G, g)[V, \nu]_{G} .
$$


By the definition of the deflation maps, we have

$$
\operatorname{Def}_{\frac{G}{G}}^{G} e_{G, g}^{G}=\frac{1}{\left|N_{G}(G, g)\right|} \sum_{\substack{(V, \nu) \in_{G} \mathcal{M}_{G}(A) \\ V \cap \Phi(G) \leq \operatorname{ker} \nu}}|V| \mu_{G}(V, \nu ; G, g)[V \Phi(G) / \Phi(G), \bar{\nu}]_{\bar{G}} .
$$

Then Equation (15) becomes

$$
\begin{aligned}
& \frac{1}{\left|N_{G}(G, g)\right|} \sum_{\substack{(V, \nu) \in_{G} \mathcal{M}_{G}(A) \\
V \cap \Phi(G) \leq k e r \nu}}|V| \mu_{G}(V, \nu ; G, g)[V \Phi(G) / \Phi(G), \bar{\nu}]_{\bar{G}} \\
& =\frac{m}{\left|N_{\bar{G}}(\bar{G}, g \Phi(G))\right|} \sum_{(W, \omega)}|W| \mu_{\bar{G}}(W, \omega ; \bar{G}, g \Phi(G))[W, \omega]_{\bar{G}} .
\end{aligned}
$$

Here $(W, \omega)$ runs over a set of representatives of the $\bar{G}$-conjugacy classes of the set $\mathcal{M}_{\bar{G}}(A)$. Now the coefficient of $[\bar{G}, 1]_{\bar{G}}$ in the right-hand side is

We also have

$$
\frac{m}{\left|N_{\bar{G}}(\bar{G}, g \Phi(G))\right|}|\bar{G}| \mu_{\bar{G}}(\bar{G}, 1 ; \bar{G}, g \Phi(G)) \text {. }
$$

$$
\mu_{\bar{G}}(\bar{G}, 1 ; \bar{G}, g \Phi(G))=\sum_{(V, \nu) \in[\bar{G}, 1]_{\bar{G}}}|V \cap g \Phi(G) O(\bar{G})| \mu(V, \bar{G}) /|V|=\frac{1}{|\bar{G}|} .
$$

Here the last equality holds since the only pair which is $\bar{G}$-conjugate to $[\bar{G}, 1]_{\bar{G}}$ is $(\bar{G}, 1)$ and hence the sum collapses to the term $|\bar{G} \cap g \Phi(G) O(\bar{G})| \mu(\bar{G}, \bar{G}) /|\bar{G}|$ and the intersection $\bar{G} \cap g \Phi(G) O(\bar{G})$ consist only of the element $g \Phi(G)$. Thus, the coefficient is

$$
\frac{m}{\left|N_{\bar{G}}(\bar{G}, g \Phi(G))\right|}=\frac{m}{|\bar{G}|}
$$

On the other hand, the coefficient of $[\bar{G}, 1]_{\bar{G}}$ on the left-hand side is

$$
\frac{1}{\left|N_{G}(G, g)\right|} \sum_{V \Phi(G)=G}|V| \mu_{G}(V, 1 ; G, g) \text {. }
$$

Since $\Phi(G)$ is the Frattini subgroup of $G$, the equality $V \Phi(G)=G$ implies that $V=G$. Then

$$
\left.\mu_{G}(G, 1 ; G, g)=\sum_{(W, 1) \in[G, 1]_{G}} \mid W \cap g O(G)\right)|\mu(W, G) /| W|=| O(G)|/| G \mid .
$$

Therefore we get

$$
m=\frac{|O(G)|}{\left|N_{G}(G, g)\right|} \cdot|G / \Phi(G)|
$$


as required.

Now let $F$ be a subfunctor of $k B^{n}$ and $G$ be a minimal group for $F$. If $G$ is not elementary abelian, then the Frattini subgroup $\Phi(G)$ of $G$ is nontrivial. Also, the coefficient $m=\frac{|O(G)|}{\left|N_{G}(G, g)\right|} \cdot|\bar{G}|$ in the previous lemma is non-zero. Indeed, all the terms $|O(G)|,|\bar{G}|$ and $\left|N_{G}(G, g)\right|$ are orders of subgroups of the $p$-group $G$ and hence $m$ is a power of $p$ and the characteristic $q$ of the field $k$ is not equal to $p$. But since the deflation of $e_{G, g}^{G}$ to a non-trivial normal subgroup is non-zero, we conclude that $G$ is not a minimal group. This proves the following result.

Proposition 4.1. Let $F$ be a subfunctor of $k B^{n}$ and $G$ be a minimal group for $F$. Then $G$ is elementary abelian.

Our next goal is to find which elementary abelian p-groups can be a minimal group of a subfunctor of $k B^{n}$. We first evaluate the deflation map on the primitive idempotents $e_{G, g}^{G}$ when $G$ is an elementary abelian $p$-group.

Proposition 4.2. Let $G$ be an elementary abelian p-group of rank $r$, let $h$ be a non-trivial element of $G$ and $H=<h>$ be the subgroup generated by $h$. Then, we have

$$
\operatorname{Def}_{G / H}^{G} e_{G, g}^{G}=m_{g, H} \cdot e_{G / H, g H}^{G / H}
$$

where

$$
m_{g, H}= \begin{cases}\frac{1-p^{r-1}}{p} & \text { if } g=1, \\ \frac{1}{p} & \text { if } g \neq 1, g \in H, \\ \frac{1-p^{r-2}}{p} & \text { if } g \notin H .\end{cases}
$$

Proof. Recall the idempotent formula

$$
e_{G, g}^{G}=\frac{1}{\left|N_{G}(G, g)\right|} \sum_{(V, \nu) \in_{G} \mathcal{M}_{G}(A)}|V| \mu_{G}(V, \nu ; G, g)[V, \nu]_{G} .
$$

Since $G$ is elementary abelian, and hence abelian, the $G$-actions seen in the formula are all trivial and hence the formula becomes

$$
e_{G, g}^{G}=\frac{1}{|G|} \sum_{(V, \nu) \in \mathcal{M}_{G}(A)}|V| \mu(V, \nu ; G, g)[V, \nu]_{G} .
$$

Furthermore, since $G$ is a non-trivial elementary abelian $p$-group, the subgroup $O(G)$ is the same as the intersection of kernels of the irreducible complex characters, and hence it is trivial. Therefore,

$$
\mu(V, \nu ; G, g)=\nu(g) \mu(V, G) /|V|
$$


and we have

$$
e_{G, g}^{G}=\frac{1}{|G|} \sum_{(V, \nu) \in \mathcal{M}_{G}(A)} \nu(g) \mu(V, G)[V, \nu]_{G} .
$$

Now we need to apply the deflation map to both sides. As in the proof of Lemma 4.1, we have

$$
\operatorname{Def}_{G / H}^{G} e_{G, g}^{G}=\frac{1}{|G|} \sum_{\substack{(V, \nu) \in \mathcal{M}_{G}(A) \\ V \cap H \leq \operatorname{ker}_{\nu}}} \nu(g) \mu(V, G)[V H / H, \bar{\nu}]_{G / H}
$$

To evaluate the right hand side of the above equality, we consider three separate cases, namely, $g=1$ or $1 \neq g \in H$ or $g \notin H$. For the first case, suppose $g=1$. Then the above equality becomes

$$
\operatorname{Def}_{G / H}^{G} e_{G, 1}^{G}=\frac{1}{|G|} \sum_{\substack{(V, \nu) \in \mathcal{M}_{G}(A) \\ V \cap H \leq \operatorname{ker}_{\nu}}} \mu(V, G)[V H / H, \bar{\nu}]_{G / H} .
$$

On the other hand, by Equation 5, the left hand side of the above equation is also equal to a multiple of

$$
e_{G / H, H}^{G / H}=\frac{1}{|G: H|} \sum_{(V, \nu) \in \mathcal{M}_{G / H}(A)} \mu(V, G / H)[V, \nu]_{G / H} .
$$

Thus, we have the equality

$$
\frac{1}{|G|} \sum_{\substack{(V, \nu) \in \mathcal{M}_{G}(A) \\ V \cap H \leq \operatorname{ker} \nu}} \mu(V, G)[V H / H, \bar{\nu}]_{G / H}=\frac{m}{|G: H|} \sum_{(W, \omega) \in \mathcal{M}_{G / H}(A)} \mu(W, G / H)[W, \omega]_{G / H} .
$$

To determine the constant $m$, we compare the coefficients. Note that the coefficient of $[G / H, 1]_{G / H}$ in the right hand side is $\frac{m}{p^{r-1}}$. In the left hand side, it is

$$
\frac{1}{|G|} \sum_{V} \mu(V, G)
$$

where $V$ runs over the subgroups satisfying $V H=G$. But, the last equality implies either that $V=G$ or that $V$ is a complement of $H$ in $G$. If $V$ is a complement of $H$, then $|V|=p^{r-1}$. But, in this case, $V$ is maximal subgroup of $G$. Thus, $\mu(V, G)=-1$. Note that there are $p^{r-1}$ many complements of $H$. If $V=G$, then obviously we have $\mu(V, G)=1$. Therefore, the coefficient in the left hand side becomes $\frac{1-p^{r-1}}{p^{r}}$. We conclude that $m=\frac{1-p^{r-1}}{p}$, as required. 
For the second case, we let $1 \neq g \in H$. As above, we have

$$
\begin{aligned}
& \frac{1}{|G|} \sum_{\substack{(V, \nu) \in \mathcal{M}_{G}(A) \\
V \cap H \leq \operatorname{ker}_{\nu}}} \nu^{-1}(g) \mu(V, G)[V H / H, \bar{\nu}]_{G / H}= \\
& \frac{m}{|G: H|} \sum_{(W, \omega)} \omega^{-1}(g H) \mu(W, G / H)[W, \omega]_{G / H} .
\end{aligned}
$$

The coefficient of $[G / H, 1]_{G / H}$ in the right hand side is again $\frac{m}{p^{r-1}}$. In the left hand side, it is equal to the sum

$$
\frac{1}{|G|} \sum_{V} \mu(V, G)
$$

where $V$ runs over subgroups containing $g$ and satisfying $V H=G$. Note that since $g \in H \cap V$ and $g \neq 1$, the subgroup $V$ cannot be a complement of $H$ and hence we must have $V=G$. Therefore the coefficient becomes $\frac{1}{p^{r}}$ and we hence we get that $m=\frac{1}{p}$, as required.

The last case where $g \notin H$ is similar to the above cases. We do not include the proof.

With this result, we can determine the subfunctors and minimal groups more explicitly. Let $\mathcal{I}=\{0\} \cup\left\{r \in \mathbb{N} \mid p^{r-1} \equiv 1(\bmod q)\right\}$ be the set of powers $r$ of $p$ for which all proper deflations by cyclic subgroups of the idempotent $e_{E, 1}^{E}$, with $E$ elementary abelian of rank $r$, is zero. We enumerate the elements of $\mathcal{I}=\left\{r_{i}\right\}_{i=0}^{\infty}$ such that $i<j$ implies $r_{i}<r_{j}$. Then we have the following theorem.

Theorem 4.1. Let $F$ be a subfunctor of $k B^{n}$ and $G$ be a minimal group of $F$. Then,

(i) the group $G$ is elementary abelian of order $p^{r}$, for some $r \in \mathcal{I}$,

(ii) the $k$-vector space $F(G)$ is 1-dimensional generated by $e_{G, 1}^{G}$ and

(iii) the subfunctor $F$ is generated by $e_{G, 1}^{G}$.

Proof. By Proposition 4.1, we know that $G$ is elementary abelian. We also know that $F(G)$ is generated by idempotents of the form $e_{G, g}^{G}$. Suppose that the idempotent $e_{G, g}^{G}$ is contained in $F(G)$ for some $g \neq 1$. Then by Proposition 4.2 , we have

$$
0 \neq \operatorname{Def}_{G /<g>}^{G} e_{G, g}^{G}=\frac{1}{p} \cdot e_{G /<g>,<g>}^{G /<g>} \in F(G /<g>) .
$$

But $G$ is minimal and hence $F(G /<g>)=0$, contradiction. Therefore, the idempotent $e_{G, 1}^{G}$ must generate $F(G)$. Moreover, if $G$ has rank $r$, then, by Proposition 4.2 .

$$
\operatorname{Def}_{G /<g>}^{G} e_{G, 1}^{G}=\frac{1-p^{r-1}}{p} \cdot e_{G /<g>,<g>}^{G /<g>} \in F(G /<g>) .
$$


Thus, we must have $p^{r-1} \equiv 1 \bmod q$. Thus we have proved the first two parts of the theorem.

To prove the last part, let $G=E_{r}$ be an elementary abelian group of rank $r$ and suppose, for a contradiction, that the idempotent $e_{E_{r}, 1}^{E_{r}}$ does not generate $F$ and let $K$ denote the subfunctor of $F$ generated by $e_{E_{r}, 1}^{E_{r}}$. Then, there exists a group $T$ such that for some element $x \in F(T)$, we have $x \notin K(T)$. Suppose $T$ has minimal order with respect to the property that $F(T) \neq K(T)$. Since $F(T)$ is an ideal of $k B^{n}(T)$, it is generated by a set $I$ of primitive idempotents. Thus, in the primitive idempotent basis, we have

$$
x=\sum_{I} x_{H, h}^{T} \cdot e_{H, h}^{T}
$$

for some constants $x_{H, h}^{T} \in k$. This implies that for some pair $(H, h)$, the idempotent $e_{H, h}^{T}$ is not contained in $K(T)$.

To determine the set $I$, suppose that $e_{H, h}^{T} \notin K(T)$ and $H \neq T$. Then, by the minimality of $T$, we have $\operatorname{Res}_{H}^{T} e_{H, h}^{T} \in K(H)$. So, for some $X \in k B^{n}\left(H \times E_{r}\right)$, we have

$$
\operatorname{Res}_{H}^{T} e_{H, h}^{T}=X \cdot e_{E_{r}, 1}^{E_{r}} .
$$

Thus, multiplying both sides by an induction biset, we get

$$
\operatorname{Ind}_{H}^{T} \operatorname{Res}_{H}^{T} e_{H, h}^{T}=\left(\operatorname{Ind}_{H}^{T} X\right) e_{E_{r}, 1}^{E_{r}} .
$$

But note that

$$
e_{H, h}^{T} \cdot \operatorname{Ind}_{H}^{T} \operatorname{Res}_{H}^{T} e_{H, h}^{T}=\alpha \cdot e_{H, h}^{T}
$$

for some non-zero $\alpha \in k$. Thus, we have

$$
e_{H, h}^{T} \cdot\left(\left(\operatorname{Ind}_{H}^{T} X\right) e_{E_{r}, 1}^{E_{r}}\right)=\alpha \cdot e_{H, h}^{T}
$$

which implies that $e_{H, h}^{T}=\frac{1}{\alpha} \cdot\left(\widetilde{e_{H, h}^{T}} \cdot \operatorname{Ind}_{H}^{T} X\right) \cdot e_{E_{r}, 1}^{E_{r}}$. This is a contradiction since we assumed that $e_{H, h}^{T}$ is not in $K(T)$. So we must have $H=T$.

Next suppose that $e_{T, t}^{T} \notin K(T)$ and the Frattini subgroup $\Phi(T)$ is non-trivial. Then, again, by the minimality of $T$, we have $\operatorname{Def}_{T / \Phi(T)}^{T} e_{T, t}^{T}=X \cdot e_{E_{r}, 1}^{E_{r}}$ for some $X \in k B^{n}(T / \Phi(T) \times T)$. Note that

$$
e_{T, t}^{T} \cdot\left(\operatorname{Inf}_{T / \Phi(T)}^{T} \operatorname{Def}_{T / \Phi(T)}^{T} e_{T, t}^{T}\right)=\beta \cdot e_{T, t}^{T}
$$

for some non-zero $\beta \in k$. Thus, we have

$$
e_{T, t}^{T} \cdot\left(\operatorname{Inf}_{T / \Phi(T)}^{T} X \cdot e_{E_{r}, 1}^{E_{r}}\right)=\beta \cdot e_{T, t}^{T}
$$

which implies $e_{T, t}^{T}=\frac{1}{\beta} \cdot\left(\widetilde{e_{T, t}^{T}} \cdot \operatorname{Inf}_{T / \Phi(T)}^{T} X\right) \cdot e_{E_{r}, 1}^{E_{r}}$ which is again a contradiction. So, we must have $\Phi(T)=1$ and $T$ is elementary abelian. 
Finally, suppose $e_{T, t}^{T} \notin K(T)$ and $T$ is elementary abelian. By part (i), the rank of $T$ is greater than or equal to that of $G$ and hence there is a subgroup $U$ of $T$ isomorphic to $G$. Without loss of generality, suppose $U=G$. Now if $t \neq 1$, then we have

$$
\operatorname{Def}_{T /<t>}^{T} e_{T, t}^{T}=\frac{1}{p} \cdot e_{T /<t>,<t>}^{T /<t>}
$$

and by similar arguments, we again obtain a contradiction. Therefore, we must have $t=1$. Also, if $P \leq R$ are elementary abelian, then the idempotent $e_{R, 1}^{R}$ is a summand of $\operatorname{Inf}_{P}^{R} e_{P, 1}^{P}$. In particular, the idempotent $e_{T, 1}^{T}$ is a summand of $\operatorname{Inf}_{E_{r}}^{T} e_{E_{r}, 1}^{E_{r}}$ and hence in $K(T)$, a contradiction. This completes the proof of the theorem.

The following corollary follows immediately.

Corollary 4.1. The A-fibered Burnside functor $k B^{n}$ over p-groups is uniserial.

Proof. Let $K$ and $L$ be subfunctors of $k B^{A}$ with the respective minimal groups $E$ and $D$. Suppose, without loss of generality, that the rank $s$ of $E$ is less than the rank $r$ of $D$. Then by the previous theorem, $K$ (resp. L) is generated by $e_{E, 1}^{E}\left(\operatorname{resp} . e_{D, 1}^{D}\right)$. We claim that $L \subset K$. To prove this, it is sufficient to show that $L(D) \subset K(D)$. But as remarked in the proof of the previous theorem, the idempotent $e_{D, 1}^{D}$ is a summand of $\operatorname{Inf}_{E}^{D} e_{E, 1}^{E}$ and since $K(D)$ is an ideal of $k B^{A}(D)$, we have $e_{D, 1}^{D} \in K(D)$. Therefore, by the previous theorem, $L(D) \subset K(D)$.

Finally we identify the subfunctors and the composition factors of the fibered Burnside functor over $p$-groups. Our description is in terms of the well-known subfunctor of intersection kernels, defined as follows. Let $F$ be a fibered biset functor and $\mathcal{H}$ be a set of minimal groups of $F$. Then, the $k$-module $K_{\mathcal{H}}^{F}(G)$ given by

$$
K_{\mathcal{H}}^{F}(G)=\bigcap_{\substack{H \\ H \in \mathcal{H}}} \operatorname{ker}(X: F(G) \rightarrow F(H))
$$

together with the induced actions of fibered bisets is a subfunctor of $F$ (cf. [4, Section 11]).

Proposition 4.3. Let $K_{0}$ denote $k B^{A}$. For $i \geq 0$, define $K_{i+1}$ recursively as follows. Let $H_{i}$ be the minimal group of $K_{i}$, and put $K_{i+1}=K_{\left\{H_{i}\right\}}^{K_{i}}$. Then,

(i) the subfunctor $K_{i+1}$ is the unique maximal subfunctor of $K_{i}$,

(ii) the minimal group $H_{i}$ of the subfunctor $K_{i}$ is the $i$-th element $E_{r_{i}}$ of the set $\mathcal{I}$ defined above.

Proof. Note that $K_{i+1}$ is a subfunctor by definition. To see that it is maximal in $K_{i}$, let $F \subset K_{i}$ be a proper subfunctor. We need to show that for any group $G$, we have $F(G) \subseteq K_{i+1}(G)$. To prove this inclusion, it suffices to show that $F\left(H_{i}\right)=0$. 
Indeed, let $x \in F(G)$ be an arbitrary element. Then, for any $A$-fibered $\left(H_{i}, G\right)$-biset ${ }_{H_{i}} X_{G}$, we have ${ }_{H_{i}} X_{G} \cdot x \in F\left(H_{i}\right)=0$. It follows that $x \in K_{H_{i}}(G)$ by the definition of $K_{i+1}$.

Now, note that we have $F\left(H_{i}\right) \subseteq K_{i}\left(H_{i}\right) \cong k \cdot e_{H_{i}, 1}^{H_{i}}$. In particular, $K_{i}\left(H_{i}\right)$ is of dimension 1 , and since $F$ is proper and $K_{i}$ is generated by $e_{H_{i}, 1}^{H_{i}}$, we must have $F\left(H_{i}\right)=0$. Indeed, otherwise $F\left(H_{i}\right)=K_{i}\left(H_{i}\right)$ and hence $F=K_{i}$. This shows that $K_{i+1}$ is the unique maximal subfunctor of $K_{i}$, completing the proof of the first part.

For the second part, since the minimal group of $K_{0}$ is the trivial group 1 which corresponds to the 0 -th element of the set $\mathcal{I}$, by part (i), it is sufficient to show that if $L \subset F$ are subfunctors of $k B^{A}$ with $L$ maximal in $F$, and if the minimal group of $F$ is $E_{r_{i}}$, then the minimal group of $L$ is $E_{r_{i+1}}$.

To prove this claim, let the minimal group of $L$ be $E_{r_{j}}$. Since $L$ is a proper subfunctor, by Theorem 4.1, we should have $i<j$. Let $K$ denote the subfunctor of $F$ generated by the idempotent $e_{E_{r_{i+1}}, 1}^{E_{E_{i+1}}}$. Then, $K$ is a proper subfunctor of $F$. Indeed, every $A$-fibered $\left(E_{r_{i}}, E_{r_{i+1}}\right)$-biset decomposes as in Theorem 2.1. However, the image of $e_{E_{r_{i+1}}, 1}^{E_{r_{i+1}}}$ under the restriction and the deflation maps are zero. Thus, we have $K\left(E_{r_{i}}\right)=0$. Now, $L$ being maximal guaranties that we have $K\left(E_{r_{i+1}}\right) \subseteq L\left(E_{r_{i+1}}\right)$. Since $K\left(E_{r_{i+1}}\right)$ is non-zero, we conclude that $j \leq i+1$ which implies $j=i+1$.

Thus we have shown that for each $r_{i} \in \mathcal{I}$, there is a subfunctor, namely $K_{i}$, of $k B^{A}$. Next we examine the set $\mathcal{I}=\{0\} \cup\left\{r \in \mathbb{N} \mid p^{r-1} \equiv 1(\bmod q)\right\}$ more closely.

If $q=0$, then clearly we have $\mathcal{I}=\{0,1\}$. If $q \neq 0$, then $\mathcal{I}$ consists of all positive integers congruent to 1 modulo $s$ where $s$ is the order of $p$ modulo $q$. Note further that if $q$ divides $p-1$, then the order $s$ is equal to 1 and $\mathcal{I}$ consists of all positive integers. Now we are ready to state our main theorem.

Theorem 4.2. Let $A$ be a cyclic p-group and $k$ be a sufficiently large field of characteristic $q$ with $q \neq p$. Then, the A-fibered Burnside functor $k B^{A}$ over $p$-groups is uniserial. Moreover we have

$$
\begin{array}{llrl}
k B^{A}=K_{0} \supset K_{1} \supset K_{\infty}=\{0\} & & \text { if } q=0, \\
k B^{A}=K_{0} \supset K_{1} \supset K_{2} \supset K_{3} \supset \cdots & & \text { if } q \neq 0 \text { and } q \mid p-1, \\
k B^{A}=K_{0} \supset K_{1} \supset K_{1+s} \supset K_{1+2 s} \supset \cdots & & \text { if } q \neq 0 \text { and } q \nmid p-1 .
\end{array}
$$

where the subfunctors $K_{i}$ are as defined above and for each $i$, the simple quotient $K_{i} / K_{i+1}$ is isomorphic to the simple A-fibered p-biset functor $S_{E_{r_{i}}, 1}$.

Proof. All the parts of the theorem is proved except the last claim concerning the simple composition factors. Note that the quotient $S_{i}=K_{i} / K_{i+1}$ is simple and the minimal group of the quotient is $E_{r_{i}}$. Thus it is sufficient to show that for each $i$, the $k$-vector space $S_{i}\left(E_{r_{i}}\right)$ is the trivial $k\left[E_{r_{i}}^{*} \rtimes \operatorname{Out}\left(E_{r_{i}}\right)\right]$-module. 
However, we have $S_{i}\left(E_{r_{i}}\right)=k \cdot e_{E_{r_{i}}, 1}^{E_{r_{i}}}$. Hence it suffices to show, for $\phi \in E_{r_{i}}^{*}$ and $\lambda \in \operatorname{Out}\left(E_{r_{i}}\right)$, that the effects of the fibered bisets $\operatorname{Tw}_{E_{r_{i}}, E_{r_{i}}}^{\phi}$ and $c_{E_{r_{i}}, E_{r_{i}}}^{\lambda}$ on the idempotent $e_{E_{r_{i}}, 1}^{E_{r_{i}}}$ are trivial. But by Proposition [3.4, we have

$$
\mathrm{Tw}_{E_{r_{i}}, E_{r_{i}}}^{\phi} \cdot e_{E_{r_{i}}, 1}^{E_{r_{i}}}=\phi(1) \cdot e_{E_{r_{i}}, 1}^{E_{r_{i}}}=e_{E_{r_{i}}, 1}^{E_{r_{i}}}
$$

and

$$
c_{E_{r_{i}}, E_{r_{i}}}^{\lambda} \cdot e_{E_{r_{i}}, 1}^{E_{r_{i}}}=e_{\lambda\left(E_{r_{i}}\right), \lambda(1)}^{E_{r_{i}}}=e_{E_{r_{i}}, 1}^{E_{r_{i}}}
$$

as required.

Remark 4.1. As remarked above, a simple fibered biset functor may have two nonisomorphic minimal groups. However, this is not the case for the simple functors that appear in the previous theorem. Indeed, we already know that any minimal group for a subfunctor of the fibered Burnside functor must be an elementary abelian p-group and, of a given order, there is a unique elementary abelian p-group.

Remark 4.2. Let $k$ be a field of characteristic zero and $A$ be a non-trivial cyclic p-group. Then, by the above theorem, there is a short exact sequence

$$
0 \longrightarrow S_{C_{p}, 1} \longrightarrow k B^{A} \longrightarrow S_{1,1} \longrightarrow 0
$$

of $A$-fibered p-biset functors. One can show that the simple head can be identified with the functor $k R_{A}$ of $A$-monomial characters and the quotient map can be chosen as the linearization map. Hence the above sequence becomes

$$
0 \longrightarrow S_{C_{p}, 1} \longrightarrow k B^{A} \longrightarrow k R_{A} \longrightarrow 0
$$

Recall from [6] that, in the case of p-biset functors, that is, when $A$ is trivial, the corresponding sequence is

$$
0 \longrightarrow k D \longrightarrow k B \longrightarrow k R_{\mathbb{Q}} \longrightarrow 0
$$

where $k D$ is the functor of torsion-free part of the Dade group, $B$ is the (ordinary) Burnside functor and $k R_{\mathbb{Q}}$ is the functor of rational representations. Existence of this sequence is one of the key results in the classification of endo-permutation modules. We do not know any natural construction that would match the simple A-fibered p-biset functor seen in the above short exact sequence.

\section{REFERENCES}

[1] Barker L., "Fibred permutation sets and the idempotents and units of monomial Burnside rings", J. Algebra 281 (2004) 535-566.

[2] Boltje R., "A general theory of canonical induction formulae", J. Algebra 206 (1998) 293-343.

[3] Boltje R., "Representation rings of finite groups, their species and idempotent formulae", to appear in J. Algebra. 
[4] Boltje R., Coşkun O., "Fibered Biset Functors", arXiv:1612.01117.

[5] Bouc S., "Foncteurs d'ensembles munis d'une double action", J. Algebra 183 (1996) 664-736.

[6] Bouc S., Thevénaz J., "The group of endo-permutation modules", Invent. Math 139 (2000) 275-349.

[7] Bouc S., 'The Dade group of a p-group', Invent. Math. 164 (2006), 189-231.

[8] Bouc S., Biset functors for finite groups, Lecture Notes in Math., vol.1990 Springer-Verlag, Berlin (2010).

[9] Dress A.W.M., "Contributions to theory of induced representations", Lecture Notes in Math. 342, Springer-Verlag, New York, 183-240 (1973).

[10] Dress A.W.M., "The ring of monomial representations, I. Structure theory", J. Algebra 18 (1971) 137-157.

[11] Green J.A., "Axiomatic representation theory for finite groups", J. Pure and Appl. Algebra 1 (1971) 41-771.

[12] Romero N,"On fibered biset functors with fibres order of prime and four", J. Algebra 387 (2013), 185?194.

[13] Thévenaz J., Webb P., 'The structure of Mackey functors', Trans. Amer. Math. Soc. 347 (1995), 1865-1961.

BoĞaZiÇI University Department of Mathematics BebeK İstanbul Turkey

University of California, Santa Cruz Department of Mathematics CA 95064 USA 